\title{
Influence of qubit displacements on quantum logic operations in a silicon-based quantum computer with constant interaction
}

\author{
D. I. Kamenev ${ }^{1}$, G. P. Berman ${ }^{1}$, and V. I. Tsifrinovich ${ }^{2}$ \\ ${ }^{1}$ Theoretical Division, T-13, Los Alamos National Laboratory, Los Alamos, New Mexico 87545 and \\ 2 Department of Physics, Polytechnic University, Brooklyn, New York 11201
}

\begin{abstract}
The errors caused by qubit displacements from their prescribed locations in an ensemble of spin chains are estimated analytically and calculated numerically for a quantum computer based on phosphorus donors in silicon. We show that it is possible to polarize (initialize) the nuclear spins even with displaced qubits by using Controlled NOT gates between the electron and nuclear spins of the same phosphorus atom. However, a Controlled NOT gate between the displaced electron spins is implemented with large error because of the exponential dependence of exchange interaction constant on the distance between the qubits. If quantum computation is implemented on an ensemble of many spin chains, the errors can be small if the number of chains with displaced qubits is small.
\end{abstract}

PACS numbers: 03.67.Lx, 75.10.Jm

\section{INTRODUCTION}

A promising candidate for solid-state quantum computation is the phosphorus-doped silicon. This semiconductor material is the backbone of microelectronic technology. In order for this architecture to be useful for quantum information processing, the positions of the phosphorus dopants in silicon must be controlled by utilizing, for example, a scanning tunneling microscope (STM) [1, 2, 3, 4, 15]. The STM can be used to create many identical arrays of phosphorus atoms on the surface of silicon. A large number of such arrays is required for the detection of the qubit states. The long decoherence time of the nuclear spins of the phosphorus donors in silicon makes this quantum computer attractive for quantum information processing.

Kane 6] proposed using nanoscale electronic gates to control the qubits. This technique has not yet been experimentally realized, so in this paper we consider a different architec- 
ture. In our approach, the exchange interaction between qubits is constant, and selective interactions are realized through the use of a magnetic field gradient and both microwave and radio-frequency pulses. Measurement can be implemented using optical techniques similar to those used in Refs. [7, 8, 9].

Each phosphorus atom has a nuclear spin $1 / 2$ and an electron spin $1 / 2$. There is a hyperfine interaction between both spins. The information is stored in the states of the nuclear spins. The interaction between the nuclear spins of neighboring phosphorus atoms is mediated by electron spins coupled to each other by the exchange interaction. The advantage of an architecture based on controlled phosphorus impurities in silicon are the potential scalability and the possibility of using advanced silicon-based semiconductor technology in the quantum computer design.

The typical size of an STM tip is larger than the lattice constant of silicon. Consequently, the phosphorus atoms in the lattice can be shifted from their prescribed locations by 1-4 lattice sites. Errors are generated because the Larmor frequencies of the displaced spins and the exchange interaction constant are modified. In this paper, this error is estimated analytically and calculated numerically for the protocol required to polarize the nuclear spins and for Controlled NOT gate between the electron spins of neighboring phosphorus atoms. If realized experimentally, each qubit chain in the first quantum silicon-based quantum computer will possibly contain the minimum number (two) of coupled qubits required to demonstrate basic principles of quantum computation in semiconductors. Hence, we specialize in this paper to the case of two phosphorus atoms in a chain. The unwanted displacements of the qubits seem to be inevitable in the quantum computer architecture. Therefore, our results are important for evaluating the possibility of constructing a working silicon-based quantum computer.

\section{HAMILTONIAN}

A schematic illustration of the system under consideration is given by Fig. 1. If the qubits in each chain are placed at a separation of $\sim 35 \mathrm{~nm}$ from each other, the nuclear-nuclear $\left(\sim 3.5 \times 10^{-4} \mathrm{~Hz}\right)$, nuclear-electron $(\sim 0.65 \mathrm{~Hz})$, and electron-electron $(\sim 1.2 \mathrm{kHz})$ dipoledipole interactions are small compared to the electron-electron exchange interaction $(\sim 2$ $\mathrm{MHz}$ ), so that one can neglect the dipole-dipole interactions. Since the relaxation time for 


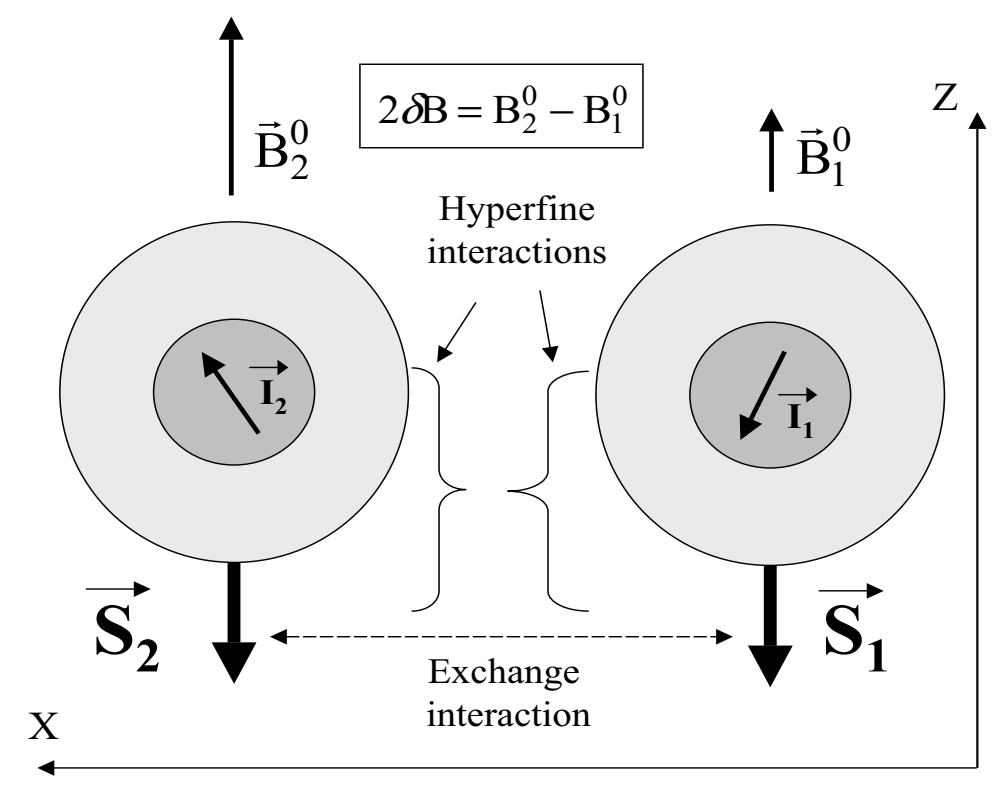

FIG. 1: A schematic illustration of two phosphorus atoms placed in a permanent magnetic field. The electron spins $\vec{S}_{1}$ and $\vec{S}_{2}$ (large arrows) of the neighboring atoms interact with each other via the exchange interaction and the nuclear spins $\vec{I}_{1}$ and $\vec{I}_{2}$ (small arrows) interact with the electron spins through the hyperfine interactions.

the electron spins at temperatures of $1-7 \mathrm{~K}$ is relatively short $(0.6-60 \mathrm{~ms}$ at $7 \mathrm{~K}[10])$, the quantum information must be stored in the states of the nuclear spins. Because the nuclear spins do not interact directly, electron spins can be used to mediate the nuclear-nuclear interactions. In this setup, the electron spins must be coherent only during the relatively short time of implementation of the quantum logic gate, such as a Controlled-NOT gate on a particular pair of qubits. The coupled electron spins of the neighboring phosphorus atoms serve as the static "quantum bus" which transfers the interaction between nearestneighbor and distant nuclear spins in a chain with more than two phosphorus atoms. In a similar technique "flying" qubits represented by photons has been proposed to mediate the qubit-qubit interaction between trapped ions [11, 12, 13, 14, 15, 16, 17].

The Hamiltonian is

$$
\hat{H}=\hat{H}^{0}+\hat{V}(t)
$$

where $\hat{H}^{0}$ is the unperturbed Hamiltonian of the system shown in Fig. 1 and $\hat{V}(t)$ is the timedependent field describing rectangular radio-frequency pulses used to implement quantum 
logic gates. The Hamiltonian $\hat{H}^{0}$ is

$$
\hat{H}^{0}=\gamma_{e} B_{1}^{z} \hat{S}_{1}^{z}+\gamma_{e} B_{2}^{z} \hat{S}_{2}^{z}-\gamma_{n} B_{1}^{z} \hat{I}_{1}^{z}-\gamma_{n} B_{2}^{z} \hat{I}_{2}^{z}+A\left(\hat{\vec{S}_{1}} \hat{\vec{I}}_{1}+\hat{\vec{S}}_{2} \hat{\vec{I}}_{2}\right)+J \hat{\vec{S}}_{1} \hat{\vec{S}}_{2}
$$

where $\hat{\vec{I}}_{k}$ and $\hat{\vec{S}}_{k}$ are the spin operators of, respectively, the $k$ th nuclear and electron spins, $k=1,2 ; \hat{I}_{k}^{z}$ and $\hat{S}_{k}^{z}$ are the projections of these operators on the $z$ axis; $\gamma_{e}$ and $\gamma_{n}$ are, respectively, the magnitudes of the electron and nuclear gyromagnetic ratios; $B_{k}^{0}$ is the permanent magnetic field at the location of the $k$ th spin; $A$ and $J$ are, respectively, the hyperfine and exchange interaction constants.

We utilized perturbation theory to calculate the eigenvalues $E_{i}, i=0,1, \ldots, 15$ of the Hamiltonian $\hat{H}^{0}$ which are given in Appendix [18]. In spite of the fact that our system allows exact analytical calculation of $E_{i}$, we prefer to use a perturbation approach because this technique allows us to separate the most important contributions $E_{i}^{(0)}$ to $E_{i}$ from the less important and to use relatively simple expressions for the eigenvalues to analyze the dynamical properties of this system. The small corrections $E_{i}^{(2)}$ are useful for calculating the parameters of the pulses in numerical simulations. The eigenvalues $E_{i}, i=1,2 \ldots, 14$ are calculated with an accuracy of $\xi^{2}(A / 2) /(2 \pi) \approx 23 \mathrm{~Hz}$, where

$$
\xi=\frac{A}{2 \gamma_{e} b} \approx 6 \times 10^{-4} .
$$

Here we assume $b=\left(B_{1}^{0}+B_{2}^{0}\right) / 2=3.3 \mathrm{~T}$, so that $\gamma_{e} b /(2 \pi)=92.5 \mathrm{GHz}, \gamma_{e} /(2 \pi)=28.025$ $\mathrm{GHz} / \mathrm{T}, A /(2 \pi)=117.53 \mathrm{MHz}[19]$.

The basis states are

$$
\left|n_{2} e_{2} e_{1} n_{1}\right\rangle
$$

where the electron spin $e_{i}$ and nuclear spin $n_{i}$ of the $i$ th phosphorus atom, $i=1,2$, can assume the values 0 and 1 ; the state $|0\rangle$ corresponds to the orientation of the spin along the direction of the permanent external magnetic field and the state $|1\rangle$ corresponds to the opposite direction. The state $|0000\rangle$ (which is not the ground state) has energy $E_{0}$, the state |0001〉 has energy $E_{1}$, etc. In the spin chain (31) there are interactions only between the neighboring spins, so this kind of spin ordering is convenient for the analysis of conditional quantum logic gates.

The basis states (3) are not the eigenstates of the Hamiltonian $\hat{H}_{0}$ because of the (offdiagonal) terms $A I_{k}^{x} S_{k}^{x}, A I_{k}^{y} S_{k}^{y}, k=1,2$, and $J S_{1}^{x} S_{2}^{x}, J S_{1}^{y} S_{2}^{y}$. However, the eigenfunctions 
approximately coincide with the basis states (3) if the conditions

$$
\epsilon=\frac{J}{2 \gamma_{e}\left(B_{2}^{z}-B_{1}^{z}\right)} \ll 1, \quad \epsilon^{\prime}=\frac{J}{\left|2 \gamma_{e}\left(B_{2}^{z}-B_{1}^{z}\right)-A\right|} \ll 1, \quad \xi \ll 1
$$

are satisfied [18]. The unwanted changes (errors) in the wave function due to the influence of the off-diagonal terms are of the order of $\epsilon$ or $\epsilon^{\prime}$ or $\xi$.

\section{IMPLEMENTATION OF QUANTUM LOGIC GATES}

The time-dependent magnetic field has the following components:

$$
\vec{B}^{1}(t)=B^{1}(\cos (\nu t+\varphi),-\sin (\nu t+\varphi), 0)
$$

where $B^{1}, \nu$, and $\varphi$ are, respectively, the amplitude, frequency and phase of the pulse, and $t$ is time. The frequency $\nu$ can assume both positive and negative values [18]. The time-dependent term in the Hamiltonian has the form

$$
\begin{gathered}
\hat{V}(t)=\left[\frac{\Omega_{e}^{0}}{2}\left(\hat{S}_{1}^{-}+\hat{S}_{2}^{-}\right)-\frac{\Omega_{n}^{0}}{2}\left(\hat{I}_{1}^{-}+\hat{I}_{2}^{-}\right)\right] e^{-i(\nu t+\varphi)}+ \\
{\left[\frac{\Omega_{e}^{0}}{2}\left(\hat{S}_{1}^{+}+\hat{S}_{2}^{+}\right)-\frac{\Omega_{n}^{0}}{2}\left(\hat{I}_{1}^{+}+\hat{I}_{2}^{+}\right)\right] e^{i(\nu t+\varphi)},}
\end{gathered}
$$

where $\Omega_{e}^{0}=\gamma_{e} B^{1}$ and $\Omega_{n}^{0}=\gamma_{n} B^{1}$.

We will consider the following three basic kinds of quantum gates: a Controlled NOT gate with the control nuclear spin and the target electron spin of the same phosphorus atom, a Controlled NOT gate with the control electron spin and the target nuclear spin, and a Controlled NOT gate between the neighboring electron spins. We now briefly describe the procedure for calculating the optimal parameters of the pulses required to implement these gates. (For a detailed analysis see Ref. [18].) Assume that the direction of the $k$ th spin in the state $|p\rangle$ is along the direction of the permanent magnetic field $\vec{B}_{k}^{0}, k=1,2$ (i.e. $\left.|p\rangle=\left|\cdots 0_{k} \cdots\right\rangle\right)$ and the state $|q\rangle=\left|\cdots 1_{k} \cdots\right\rangle$ is related to the state $|p\rangle$ by a flip of the $k$ th spin. In order to flip the $k$ th spin in the state $|p\rangle$ or in the state $|q\rangle$, the frequency $\nu$ and the time-duration $\tau$ of the rectangular pulse must satisfy the conditions

$$
\nu=E_{q}-E_{p}, \quad \tau=\frac{\Omega}{\pi},
$$

where $\Omega$ is the Rabi frequency of the pulse. 


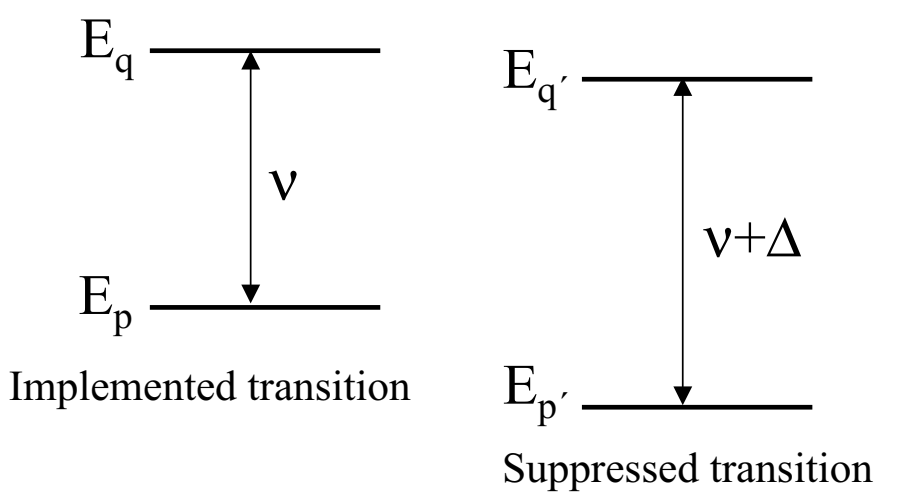

FIG. 2: A schematic illustration of the energy levels ivolved in implementation of a Controlled-NOT gate. The frequency $\nu$ is the frequency of the pulse.

Assume that we wish to suppress the transition $\left|p^{\prime}\right\rangle \leftrightarrow\left|q^{\prime}\right\rangle$, where the $k$ th spin in the state $\left|p^{\prime}\right\rangle$ is along the direction of the permanent magnetic field $\vec{B}_{k}^{0}$ and the state $\left|q^{\prime}\right\rangle$ is related to the state $\left|p^{\prime}\right\rangle$ by a flip of the $k$ th spin; $|p\rangle \neq\left|p^{\prime}\right\rangle$ and $|q\rangle \neq\left|q^{\prime}\right\rangle$. A schematic illustration of the involved energy levels and transitions is shown in Fig. 2. The Rabi frequency of the pulse must satisfy the $2 \pi K$-condition [20, 21]

$$
\Omega=\frac{|\Delta|}{\sqrt{4 K^{2}-1}}
$$

where $K=1,2, \ldots$ is an integer number, $\Omega=\Omega_{e}$ for an electron spin and $\Omega=\Omega_{n}$ for a nuclear spin, and

$$
\Delta=E_{q^{\prime}}-E_{p^{\prime}}-\nu
$$

The probability of transition generated by the pulse with the Rabi frequency $\Omega$ and detuning $\Delta$ is $[20,21]$

$$
R=\frac{\Omega^{2}}{\lambda^{2}} \sin ^{2}\left(\frac{\pi \lambda}{2 \Omega}\right),
$$

where $\lambda=\sqrt{\Omega^{2}+\Delta^{2}}$. We have $R=1$ for $\Delta=0$ and $R=0$ if $\Omega$ satisfies Eq. (8). These two conditions allow one to implement the Controlled NOT gate as indicated in Fig. 2.

\section{IV. $J$ AS A FUNCTION OF THE DISTANCE BETWEEN THE QUBITS}

A simple approximation of the exchange interaction adequate for our purposes is given by the Herring-Flicker formula [6, 22]

$$
J=1.642 \frac{e^{2}}{\kappa a_{\mathrm{B}}}\left(\frac{a}{a_{\mathrm{B}}}\right)^{5 / 2} \exp \left(-\frac{2 a}{a_{\mathrm{B}}}\right),
$$


where $e$ is the electron charge, $\kappa=11.9$ is the dielectric constant of silicon, and $a$ is the distance between the qubits. The effective Bohr radius is

$$
a_{\mathrm{B}}=\kappa \frac{M}{M^{*}} a_{\mathrm{B}}^{0} \approx 33.14 \AA,
$$

where $a_{\mathrm{B}}^{0} \approx 0.5292 \AA$ is the Bohr radius, $M$ is the electron mass, $M^{*}=0.19 M$ is the effective electron mass. In Eq. (11), $a=N a_{0}$ where $N-1$ is the number of interstitial silicon atoms between the two phosphorus atoms serving as qubits and $a_{0}=7.68 \AA$. We assume that the qubits are placed on the (100) surface of silicon and the direction of the permanent magnetic field is perpendicular to this surface. In Table I we show the values of $J /(2 \pi)$ for different $N$ calculated using Eq. (111). The controlled separation between the qubits can be varied by changing $N$. In this paper we assume that the predefined spacing (desired spacing without unwanted displacements) is $N_{0}=47$, so that the predefined exchange interaction constant is $J_{0} /(2 \pi)=1.97 \mathrm{MHz}$.

\begin{tabular}{|c|c|c|c|c|c|c|c|c|c|c|c|c|}
\hline$N$ & 40 & 41 & 42 & 43 & 44 & 45 & 46 & 47 & 48 & 49 & 50 & 51 \\
$a(\mathrm{~nm})$ & 30.72 & 31.49 & 32.26 & 33.02 & 33.79 & 34.56 & 35.33 & 36.10 & 36.86 & 37.63 & 38.40 & 39.17 \\
$J /(2 \pi)(\mathrm{MHz})$ & 33.75 & 22.58 & 15.09 & 10.07 & 6.71 & 4.465 & 2.97 & 1.97 & 1.306 & 0.865 & 0.573 & 0.37855 \\
\hline
\end{tabular}

TABLE I: The distance $a$ between the neighboring phosphorus atoms and the exchange interaction constant $J /(2 \pi)$ for different $N$, where $N-1$ is the number of interstitial silicon atoms.

\section{CONTROLLED NOT GATES BETWEEN THE ELECTRON AND NUCLEAR SPINS}

We now derive the pulse parameters of four pulses required to initialize the nuclear spins. Each pulse implements one logical gate. The gates are [18]
(a) $\mathrm{CN}_{\mathrm{n} 1, \mathrm{e} 1}$,
(b) $\mathrm{CN}_{\mathrm{e} 1, \mathrm{n} 1}$,
(c) $\mathrm{CN}_{\mathrm{n} 2, \mathrm{e} 2}$,
(d) $\mathrm{CN}_{\mathrm{e} 2, \mathrm{n} 2}$,

where $\mathrm{CN}_{i, j}$ denotes a Controlled NOT gate, $i$ is the number of the control qubit and $j$ is number of the target qubit. The protocol (13) includes also a delay time (of the order of 0.1 ms) between pulses (b) and (c) and after the pulse (d). During this time the electron spins relax to their ground states. We assume that the relaxing electron spins do not influence the corresponding nuclear spins [18]. 
In order to calculate the energies and resonant frequencies we will use the following parameters:

$$
\begin{gathered}
\frac{J / 2}{2 \pi}=0.985 \mathrm{MHz}, \frac{A / 2}{2 \pi}=58.765 \mathrm{MHz}, \quad \frac{\gamma_{e} b}{2 \pi}=92.48 \mathrm{GHz}, \quad \frac{\gamma_{n} b}{2 \pi}=56.93 \mathrm{MHz}, \\
\frac{\gamma_{e} \delta B}{2 \pi}=65.76 \mathrm{MHz}, \quad \frac{\gamma_{n} \delta B}{2 \pi}=40.48 \mathrm{kHz}
\end{gathered}
$$

where $\delta B=\left(B_{2}-B_{1}\right) / 2$. Here we assume $b=3.3 \mathrm{~T}, \gamma_{n} /(2 \pi)=17.25144 \mathrm{MHz} / \mathrm{T}$, the magnetic field gradient is $1.3 \times 10^{5} \mathrm{~T} / \mathrm{m}$, and from Table I we use $a=36.1 \mathrm{~nm}$.

We now calculate the parameters of the pulse implementing gate (a). The resonant transition is $|1101\rangle \leftrightarrow|1111\rangle$. The resonant frequency for this transition is

$$
\frac{\nu_{1}}{2 \pi} \approx \frac{E_{15}-E_{13}^{(0)}}{2 \pi}=\frac{1}{2 \pi}\left[-\gamma_{e} b+\frac{A}{2}+\frac{J}{2}+\sqrt{\left(\gamma_{e} \delta B\right)^{2}+J^{2} / 4}\right]=-92.35 \mathrm{GHz} .
$$

The near-resonant transition to be suppressed is $|1100\rangle \leftrightarrow|1110\rangle$. The detuning for this transition is

$$
\begin{gathered}
\frac{\Delta_{1}}{2 \pi} \approx \frac{E_{14}^{(0)}-E_{12}^{(0)}-\nu_{1}}{2 \pi}= \\
\frac{1}{2 \pi}\left[\sqrt{\left(\gamma_{e} \delta B-A / 2\right)^{2}+J^{2} / 4}-\sqrt{\left(\gamma_{e} \delta B\right)^{2}+J^{2} / 4}-\frac{A}{2}\right]=-117.47 \mathrm{MHz}
\end{gathered}
$$

In order to suppress the near-resonant transition with the detuning $\Delta_{i}$ the Rabi frequency of the pulse must satisfy the $2 \pi K$ condition

$$
\frac{\Omega_{i}}{2 \pi}=\frac{\left|\Delta_{i}\right|}{2 \pi \sqrt{4 K^{2}-1}}
$$

where $K$ is an integer number and $i$ is the pulse number. For the first pulse with $\Delta_{1}$ given by Eq. (16) and for $K=K_{e}=1$, we have

$$
\frac{\Omega_{1}}{2 \pi}=\frac{\left|\Delta_{1}\right|}{2 \pi \sqrt{3}}=67.82 \mathrm{MHz} .
$$

There are three sources of errors in our model: (i) nonresonant excitations, (ii) offdiagonal components of the exchange and hyperfine interactions, and (iii) qubit displacements. The error in the probability amplitude due to the nonresonant transitions is proportional to the ratio [23]

$$
\mu=\frac{\Omega}{2|\delta \omega|},
$$

where $\delta \omega$ is the difference between the frequency corresponding to the nonresonant transition and the frequency of the pulse. 


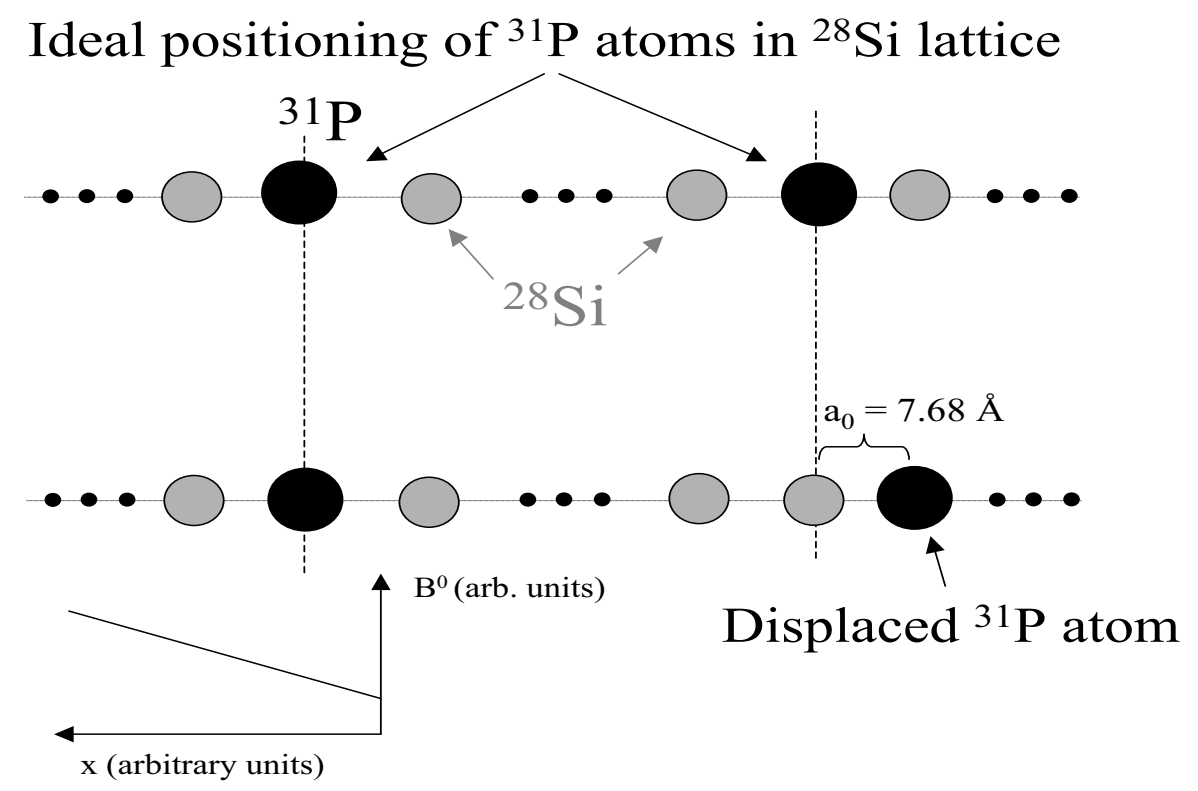

FIG. 3: A schematic illustration of an ideal qubit chain (upper part) and a qubit chain with a displaced qubit (lower part).

The order of magnitude of the error generated by a single pulse acting on an electron spin can be estimated by adding probability errors generated due to all three mechanisms (i)-(iii),

$$
P=1-R+\epsilon^{2}+\mu^{2},
$$

where $\epsilon, R$, and $\mu$ are given by Eqs. (41), (10), and (19).

We now analytically estimate the error generated by the first pulse due to the displacement of the first phosphorus atom using Eq. (10). This displacement introduces an unwanted change in the Larmor frequency of the first qubit and the correction $d J$ to the exchange interaction constant $J$. Let qubit 1 be displaced in the negative $x$ direction by one lattice site as shown in Fig. [3 From Table I we find

$$
\frac{d J}{2 \pi}=1.306 \mathrm{MHz}-1.97 \mathrm{MHz}=-0.664 \mathrm{MHz} .
$$

The permanent magnetic field at the location of the first qubits is $B_{1}^{0}-d B$, where

$$
d B=\frac{B_{2}^{0}-B_{1}^{0}}{N} \approx 9.985 \times 10^{-5} \mathrm{~T} .
$$

From Eq. (15) we have

$$
\nu_{1} \approx-\gamma_{e} B_{1}^{0}+\frac{A}{2}+\frac{J}{2}
$$




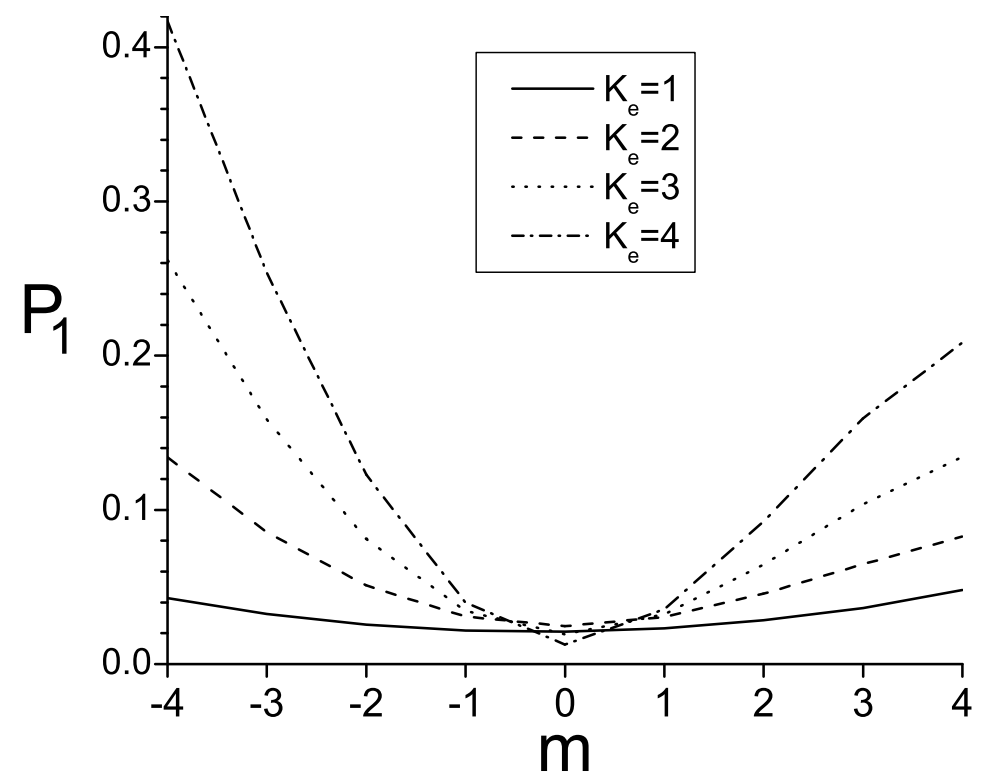

FIG. 4: Probability error $P_{1}$ generated by gate (a) in Eq. (13) as a function of displacement $m$ of the first phosphorus atom for four values of $K_{e}$.

so that the unwanted detuning is

$$
\frac{\Delta_{1}^{\prime}(m=-1)}{2 \pi} \approx \frac{1}{2 \pi}\left(\gamma_{e} d B+\frac{\delta J}{2}\right)=2.466 \mathrm{MHz},
$$

where $\gamma_{e} d B /(2 \pi)=2.798 \mathrm{MHz}$ and we used Eqs. (21) and (22). If we put $\Delta_{1}^{\prime}$ instead of $\Delta$ and $\Omega_{1}$ instead of $\Omega$ in Eq. (10) we find the probability error $1-R$ due to the unwanted qubit displacement.

For displacement in the opposite direction with $m=1$

$$
\frac{\Delta_{1}^{\prime}(m=1)}{2 \pi} \approx \frac{1}{2 \pi}\left(-\gamma_{e} d B+\frac{\delta J^{\prime}}{2}\right)=-1.8 \mathrm{MHz}
$$

where $\delta J^{\prime} /(2 \pi)=1 \mathrm{MHz}$ (see Table I). Since $\left|\Delta_{1}^{\prime}(m=1)\right|<\left|\Delta_{1}^{\prime}(m=-1)\right|$, the error $P_{1}$ for the displacement in the negative direction along the $x$ axis is larger than the error for displacement in the positive direction because $1-R$ in Eq. (201) increases with $\left|\Delta_{1}^{\prime}\right|$ increasing.

In Fig. 4 we plot the error $P_{1}$ for the transition $|1101\rangle \rightarrow|1111\rangle$ generated by the first pulse for the chain with displaced qubit 1 as a function of the qubit displacement $m$ along the $x$ axis. This plot is asymmetric with respect to $m=0$ which follows from the analysis presented in the previous paragraph. The data are obtained by utilizing the eigenfunctions of full Hamiltonian in the rotating frame. (For a description of the numerical solution 


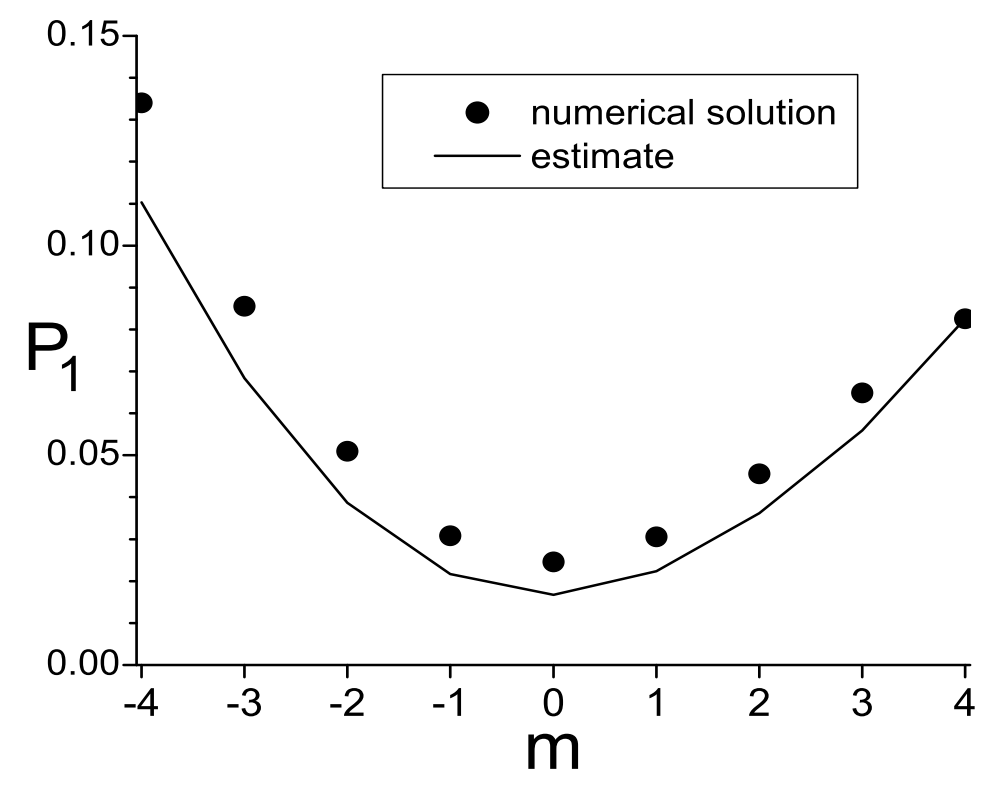

FIG. 5: Probability error $P_{1}$ generated by by gate (a) in Eq. (13) as a function of displacement $m$ of the first phosphorus atom along the $x$ axis for $K_{e}=2$. The estimate is calculated using Eq. (20).

see Ref. [18].) The parameters of the pulses in our numerical simulations are calculated analytically using the eigenvalues from the Appendix. In the figure, $m$ is the number of lattice sites for a displaced qubit, so that the displacement is $m a_{0}, m=-4,-3, \ldots, 4$. Our simulations (not presented here) show that this and other pulses generate the maximum error in situation when the "active" qubit to be flipped is displaced and the error is smaller when the neighboring qubit is displaced.

Since $\left|\Delta_{1}^{\prime}\right| \ll \Omega_{1}$ [see Eqs. (18), (23), and (24)] the error $P_{1}$ is relatively small small. (Here and below we assume that the error $P$ is small if $P \ll 1$.) The smallness of the error generated by the first pulse is due to (a) the neighboring phosphorus atoms being located relatively far from each other; (b) the electron Rabi frequency being relatively large, and (c) the exchange interaction constant $A$, responsible for the Controlled NOT gate, not being modified by the displacements of the phosphorus atoms.

From Fig. 4, one can see that the error increases as $|m|$ increases. As follows from this figure, the probability error becomes more sensitive to the qubit displacement when $K_{e}$ becomes relatively large and the Rabi frequency becomes relatively small. In Fig. [5 the probability error estimate (20) is compared with the results of the numerical solution. One can see that our estimate is satisfactory and supports the approach taken. The asymmetry of the plot in Fig. [5 with respect to $m=0$ is of the same origin as that in Fig. 4 discussed 
before.

We now estimate the error associated with a flip of the first nuclear spin in the situation when the first phosphorus atom is shifted from its ideal position to $m=-1$. We first calculate the pulse parameters. The resonant transition is $|1111\rangle \leftrightarrow|1110\rangle$. The frequency of this transition is

$$
\frac{\nu_{2}}{2 \pi} \approx \frac{E_{15}-E_{14}^{(0)}}{2 \pi}=\frac{1}{2 \pi}\left(\gamma_{n} b-\gamma_{n} \delta B+\frac{A}{2}\right)=115.655 \mathrm{MHz} .
$$

The near-resonant transition to be suppressed is $|1100\rangle \leftrightarrow|1101\rangle$. One can show that the detuning for this transition is $\Delta_{2}=\Delta_{1}$, where $\Delta_{1}$ is given by Eq. (16). The $2 \pi K$ condition for the second pulse reads

$$
\frac{\Omega_{2}}{2 \pi}=\frac{\left|\Delta_{2}\right|}{2 \pi \sqrt{4 K_{n}^{2}-1}}
$$

where $K_{n}$ is an integer. In order to suppress the flip of nuclear spin 2 , the value of $\Omega_{2}$ must be much smaller than the frequency difference associated with flip of the second nuclear spin. The unwanted off-resonant transition is $|0110\rangle \leftrightarrow|1110\rangle$. The detuning for this transition is $\delta \omega=2 \gamma_{n} \delta B$. From Eq. (19) and for $\mu \ll 1$, we obtain the condition

$$
K_{n} \gg 363
$$

or $\Omega_{2} /(2 \pi) \ll 162 \mathrm{kHz}$.

We now calculate the error associated with displacement of the first phosphorus atom for $m=-1$. The corrections to the magnetic field is given by Eq. (22). The correction to $\gamma_{n} \delta B /(2 \pi)$ is $0.86 \mathrm{kHz}$ and the correction to $\gamma_{n} b /(2 \pi)$ is $-0.86 \mathrm{kHz}$. From Eq. (25) the unwanted detuning from the resonance is

$$
\frac{\Delta_{2}^{\prime}}{2 \pi}=-1.72 \mathrm{kHz}
$$

From the condition $\Omega_{2} \gg\left|\Delta_{2}^{\prime}\right|$, we find $\Omega_{2} \gg 1.3 \mathrm{kHz}$, or

$$
K_{n} \ll 34,148
$$

From Eqs. (27) and (28), one can see that it is possible to make both the errors due to the nonresonant excitations and the errors due to the unwanted displacements small.

In Fig. 6] we plot the error as a function of dimensionless displacement $m$ for four different values of $K_{n}$. This plot has the same features as Fig. 4(a). The probability error $P_{2}$ is 


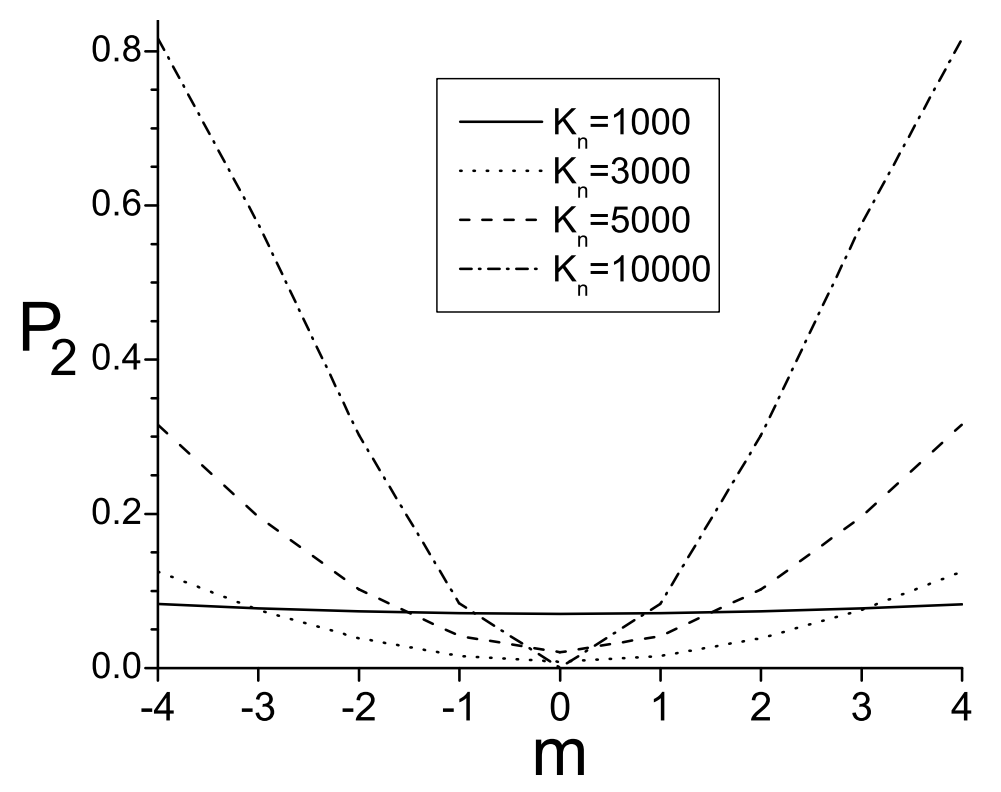

FIG. 6: The probability error $P_{2}$ generated by the second pulse on the first nuclear spin as a function of displacement $m$ of the first phosphorus atom along the $x$ axis for five values of $K_{n}$. The data are obtained by numerical solution of the full Hamiltonian (1).

independent of the direction of the displacement because $P_{2}$ depends only on the magnitude of the deviation of magnetic field from the optimal value in the location of the qubit. The error due to the deviation of $J$ from the optimal value $J_{0}$ does not contribute to $P_{2}$ because the first nuclear spin does not directly interact with the second electron spin and second nuclear spin. Since the nuclear spins do not interact with each other and electron and nuclear spins of different phosphorus atoms do not interact with each other, the displacement of the second phosphorus atom practically does not affect the quantum logic operations on the nuclear spin of the first atom. This was also confirmed by our calculations (not presented here). This result follows also from Eq. (25), where $\nu_{2}=\gamma_{n} B_{1}^{0}+A / 2$ is independent of $J$ and $B_{2}^{0}$.

\section{ERROR IN THE INITIALIZATION ALGORITHM}

We have analyzed the errors generated by the first and second pulses. The errors generated by the third and fourth pulses [pulses (c) and (d) in Eq. (13)] are of the same order of magnitude. As follows from these results, one can make the error relatively small and polarize (initialize) the nuclear spins even in the situation when the phosphorus atoms are 


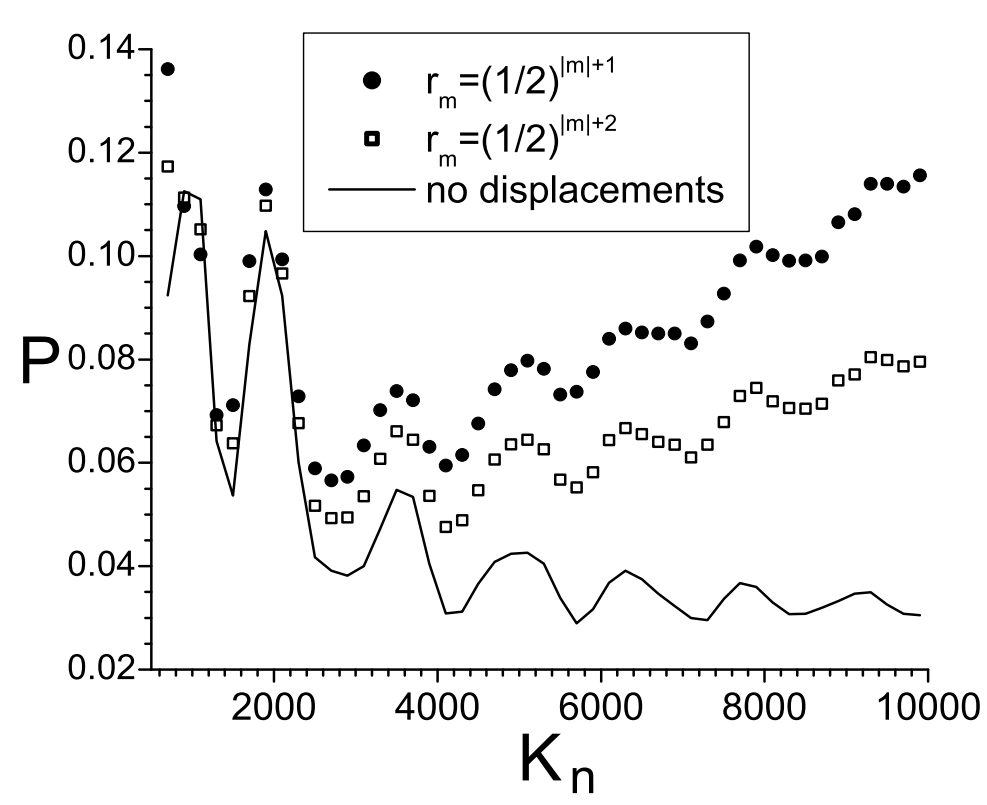

FIG. 7: The probability of error $P=1-\left|C_{15}(T)\right|^{2}$ generated by the initialization protocol as a function of $K_{n}$. Each phosphorus atom in an ensemble of 10,000 spin pairs had the probability $r_{m}$, $m=-4,-3, \ldots, 3,4$ to be displaced in a random direction along the $x$ axis. For a perfect sample without displacements $r_{m}=0$ (solid line), $K_{e}=1$. The data are obtained by numerical solution of the full Hamiltonian (1).

displaced from their prescribed locations in the chains. To illustrate this feature we modeled the initialization protocol on an ensemble of 10,000 noninteracting spin chains. Each chain consisted of two phosphorus atoms. The initial state for each chain was the superposition

$$
C_{6}(0)|0110\rangle+C_{7}(0)|0111\rangle+C_{14}(0)|1110\rangle+C_{15}(0)|1111\rangle
$$

with randomly chosen normalized complex coefficients $C_{i}(t), i=6,7,14,15$. The probability of error, $P=1-\left|C_{15}(T)\right|^{2}$, where $T$ is the time of implementation of the initialization algorithm, as a function of $K_{n}$ is presented in Fig. 17. We calculated three situations. In the first situation each phosphorus atom in the ensemble had the probability $r_{m}=(1 / 2)^{|m|+1}$, $m=-4,-3, \ldots, 3,4$ to be displaced in a random direction along the $x$ axis (filled circles in Fig. (7). In the second situation the probability of displacement is $r_{m}=(1 / 2)^{|m|+2}$ which corresponds to a sample of higher quality. For comparison, for the third situation we plot $P$ for a perfect sample without displacements $\left(r_{m}=0\right)$. The data in Fig. [7 are averaged over 40 realizations with different initial conditions and different sets of random displacements. The size of the error bars is of the order of the size of the symbols. The Rabi frequencies 
corresponding to different values of $K_{n}$ can be obtained from Eq. (26) with $\left|\Delta_{2}\right| /(2 \pi) \approx$ $\left|\Delta_{4}\right| /(2 \pi) \approx 117.5 \mathrm{MHz}$. If $K_{n}$ assumes the values in the interval from 700 to 10,000 , the magnitudes of $\Omega_{2}$ and $\Omega_{4}$ vary in the interval from $84 \mathrm{kHz}$ to $5.9 \mathrm{kHz}$.

As follows from Fig. 6, when $K_{n}$ is relatively small (and the Rabi frequency is relatively large) the error is mostly determined by the off-resonant excitations and only slightly depends on the quality of the sample. For relatively large values of $K_{n}$ (and small Rabi frequencies) the error is smaller for better samples with smaller number of displacements.

\section{CONTROLLED NOT GATE BETWEEN ELECTRON SPINS}

As follows from the results presented above, one can make the error in the Controlled NOT gates between the nuclear and electron spins of the same phosphorus to be relatively small because the coupling constant $A$ responsible for the Controlled NOT gates is not modified by the displacements. Now we will consider the error generated by the Controlled NOT gate between the electron spins of the neighboring phosphorus atoms. One can expect this error to be relatively large because a displacement modifies the coupling constant $J$ which is the key parameter for this gate.

The detuning is $\left|\Delta_{e}\right| \approx J$, so that the Rabi frequency is equal to

$$
\Omega_{e}=\frac{\left|\Delta_{e}\right|}{\sqrt{4 K_{e}^{\prime 2}-1}} \approx \frac{J}{\sqrt{4 K_{e}^{\prime 2}-1}}
$$

where $K_{e}^{\prime}=1,2, \ldots$ is an integer number. From Eq. (11) we can find the modification $\delta J=J-J_{0}$ from the "ideal" value $J_{0}$ due to the displacement $a_{0} m=7.68 \mathrm{~m} \AA$,

$$
\delta J \approx J_{0}\left[\frac{5 m}{2 N}+e^{2 a_{0} m / a_{\mathrm{B}}}-1\right]
$$

For $N=47$ and $a_{B}=33.14 \AA$, we obtain

$$
\delta J / J_{0}=0.4103 m+0.1082 m^{2}+0.0166 m^{3}+0.0019 m^{4}+\ldots
$$

For the minimum possible displacement with $m=-1$, we have $\delta J / J_{0} \approx-0.32$ which can be verified from data in Table I.

The unwanted modification of the Rabi frequency (29) caused by the displacement is

$$
\delta \Omega_{e}=\frac{\delta J}{\sqrt{4 K_{e}^{\prime 2}-1}}=-0.32 \Omega_{e} .
$$


The error is small if two conditions are satisfied: (i) $\left|\delta \Omega_{e}\right| \ll \Omega_{e}$ and (ii) modulus of the unwanted detuning $\left|\Delta_{e}^{\prime}\right|$ is small, $\left|\Delta_{e}^{\prime}\right| \ll \Omega_{e}$. One can show that the unwanted detuning from the resonance caused by the displacement is $\left|\Delta_{e}^{\prime}\right| \approx \delta J / 2$, so that both conditions (i) and (ii) cannot be satisfied by any choice of parameters of the system and the error is large. We checked this result numerically and determined that the error $P_{e}$ is close to unity for $|m| \neq 0$ and $\left|P_{e}\right| \sim 10^{-4}$ to $10^{-3}$ for $m=0$.

We now estimate the error if one qubit is displaced in a spin chain with more than two phosphorus atoms. The presented above analysis is valid for the edge qubits of this chain. Consider, for example, the state $\left|\cdots 1_{k+1} 1_{k} 1_{k-1} \cdots\right\rangle$ formed by coupled electron spins. (Here we do not indicate the states of the nuclear spins.) If the the $k$ th atom is not displaced, then the frequency resonant for the transition of the $k$ th qubit is

$$
\frac{\nu_{e}}{2 \pi}=\frac{1}{2 \pi}\left(\frac{J_{0}}{2}+\frac{J_{0}}{2}+\ldots\right)=\frac{J_{0}}{2 \pi}+\ldots
$$

where $J_{0} /(2 \pi)=1.97 \mathrm{MHz}$. If the $k$ th qubit is displaced in any direction along the chain by one lattice cite then instead of the term $J_{0} /(2 \pi)$ in the right-hand side of Eq. (31) we have from Table I

$$
\frac{1}{2 \pi}\left[\frac{J(m=1)}{2}+\frac{J(m=-1)}{2}\right]=2.14 \mathrm{MHz},
$$

so that the unwanted detuning from the resonance is

$$
\Delta_{e}^{\prime}=0.086 J_{0}
$$

From Eq. (29) for $K_{e}^{\prime}=1$, we obtain

$$
\Omega_{e}=\frac{J_{0}}{\sqrt{3}} \approx 0.58 J_{0}
$$

From Eqs. (32) and (33) one can see that the unwanted detuning is relatively small $\left|\Delta_{e}^{\prime}\right| \ll \Omega_{e}$ and condition (ii) is satisfied. One can show that this condition is not satisfied for the transitions $\left|\cdots 1_{k+1} 1_{k} 0_{k-1} \cdots\right\rangle \leftrightarrow\left|1_{k+1} 0_{k} 0_{k-1}\right\rangle$. The deviation in Rabi frequency [condition (i)] is always relatively large. For example, if the target $k$ th qubit is displaced in the negative direction $(\mathrm{m}=-1)$ and the $(k-1)$ th qubit is the control qubit, then $\delta \Omega_{e}$ is given by Eq. (30) and condition $\left|\delta \Omega_{e}\right| \ll \Omega_{e}$ is not satisfied, so that the Controlled NOT gate between the displaced electron spins generate large error.

Presented here analysis demonstrate that the Controlled NOT gate between the electron spins cannot be performed on displaced qubits. If quantum computation is implemented 
on an ensemble of many spin chains, the errors can be small if the number of chains with displaced qubits is small.

In conclusion, we note that the problem of unwanted displacements appears only in quantum computation performed on an ensemble of spin chains. If one works with one chain only, one can measure the positions of the phosphorus atoms using a scanning tunneling microscope and optimize the parameters of the pulses appropriately.

\section{Acknowledgments}

We thank M. E. Hawley, G. W. Brown, and G. D. Doolen for useful discussions. This work was carried out under the auspices of the National Nuclear Security Administration of the U.S. Department of Energy at Los Alamos National Laboratory under Contract No. DE-AC52-06NA25396.

\section{APPENDIX}

The eigenvalues of the system of two phosphorus atoms in a permanent magnetic field are

$$
\begin{gathered}
E_{0}=\left(\gamma_{e}-\gamma_{n}\right) b+\frac{A}{2}+\frac{J}{4}, \\
E_{1}^{(0)}=\gamma_{e} b-\gamma_{n} \delta B+\frac{J}{4}, \quad E_{1}^{(2)}=\frac{A^{2}}{4\left[E_{1}^{(0)}-E_{2}^{(0)}\right]}, \\
E_{2}^{(0)}=-\gamma_{n} b-\frac{J}{4}+\sqrt{\left(\gamma_{e} \delta B\right)^{2}+\frac{J^{2}}{4}}, \quad E_{2}^{(2)}=-E_{1}^{(2)}, \\
E_{3}^{(0)}=-\gamma_{n} \delta B-\frac{J}{4}+\sqrt{\left(\gamma_{e} \delta B+\frac{A}{2}\right)^{2}+\frac{J^{2}}{4}}, \quad E_{3}^{(2)}=0, \\
E_{4}^{(0)}=-\gamma_{n} b-\frac{J}{4}-\sqrt{\left(\gamma_{e} \delta B\right)^{2}+\frac{J^{2}}{4}, \quad E_{4}^{(2)}=\frac{A^{2}}{4\left[E_{4}^{(0)}-E_{8}^{(0)}\right]},} \\
E_{5}^{(0)}=-\gamma_{n} \delta B-\frac{J}{4}-\sqrt{\left(\gamma_{e} \delta B+\frac{A}{2}\right)^{2}+\frac{J^{2}}{4}}, \\
E_{5}^{(2)}=\frac{A^{2}}{4}\left[\frac{1}{E_{5}^{(0)}-E_{6}^{(0)}}+\frac{1}{E_{5}^{(0)}-E_{9}^{(0)}}\right], \\
E_{6}^{(0)}=-\left(\gamma_{e}+\gamma_{n}\right) b-\frac{A}{2}+\frac{J}{4}, \quad E_{6}^{(2)}=\frac{A^{2}}{4}\left[\frac{1}{E_{6}^{(0)}-E_{5}^{(0)}}+\frac{1}{E_{6}^{(0)}-E_{10}^{(0)}}\right],
\end{gathered}
$$




$$
\begin{gathered}
E_{7}^{(0)}=-\gamma_{e} b-\gamma_{n} \delta B+\frac{J}{4}, \quad E_{7}^{(2)}=\frac{A^{2}}{4\left[E_{7}^{(0)}-E_{11}^{(0)}\right]}, \\
E_{8}^{(0)}=\gamma_{e} b+\gamma_{n} \delta B+\frac{J}{4}, \quad E_{8}^{(2)}=-E_{4}^{(2)}, \\
E_{9}^{(0)}=\left(\gamma_{e}+\gamma_{n}\right) b-\frac{A}{2}+\frac{J}{4}, \quad E_{9}^{(2)}=\frac{A^{2}}{4}\left[\frac{1}{E_{9}^{(0)}-E_{5}^{(0)}}+\frac{1}{E_{9}^{(0)}-E_{10}^{(0)}}\right], \\
E_{10}^{(0)}=\gamma_{n} \delta B-\frac{J}{4}+\sqrt{\left(\gamma_{e} \delta B-\frac{A}{2}\right)^{2}+\frac{J^{2}}{4}}, \\
E_{10}^{(2)}=\frac{A^{2}}{4}\left[\frac{1}{E_{10}^{(0)}-E_{6}^{(0)}}+\frac{1}{E_{10}^{(0)}-E_{9}^{(0)}}\right] \\
E_{11}^{(0)}=\gamma_{n} b-\frac{J}{4}+\sqrt{\left(\gamma_{e} \delta B\right)^{2}+\frac{J^{2}}{4}, \quad E_{11}^{(2)}=-E_{7}^{(2)},} \\
E_{12}^{(0)}=\gamma_{n} \delta B-\frac{J}{4}-\sqrt{\left(\gamma_{e} \delta B-\frac{A}{2}\right)^{2}+\frac{J^{2}}{4}}, \quad E_{12}^{(2)}=0, \\
E_{13}^{(0)}=\gamma_{n} b-\frac{J}{4}-\sqrt{\left(\gamma_{e} \delta B\right)^{2}+\frac{J^{2}}{4}}, \quad E_{13}^{(2)}=\frac{A^{2}}{4\left[E_{13}^{(0)}-E_{14}^{(0)}\right]}, \\
E_{14}^{(0)}=-\gamma_{e} b+\gamma_{n} \delta B+\frac{J}{4}, \quad E_{14}^{(2)}=-E_{13}^{(2)}, \\
E_{15}=\left(-\gamma_{e}+\gamma_{n}\right) b+\frac{A}{2}+\frac{J}{4},
\end{gathered}
$$

where $b=\left(B_{2}^{0}+B_{1}^{0}\right) / 2$ and $\delta B=\left(B_{2}^{0}-B_{1}^{0}\right) / 2$. In the text we assume $E_{i}=E_{i}^{(0)}+E_{i}^{(2)}$. The eigenvalues $E_{10}$ and $E_{12}$ are presented for the case $A / 2 \leq \gamma_{e} \delta B$. For the opposite case, $A / 2>\gamma_{e} \delta B$, one must exchange the eigenvalues $E_{10} \leftrightarrow E_{12}$.

[1] J. W. Lyding, G. C. Abeln, T.-C. Shen, C. Wang, and J. R. Tucker, J. Vac. Sci. Technol. B 12, 3735 (1994).

[2] D. P. Adams, T. M. Mayer, and B. S. Swartzentruber, J. Vac. Sci. Technol. B 14, 1642 (1996).

[3] C. Thirstrup, M Sakurai, T. Nakayama, M. Aono, Surf. Sci. 411, 203 (1998).

[4] J. R. Tucker, and T.-C. Shen, Solid-State Electronics 42, 1061 (1998).

[5] J. L. O’Brien, S. R. Schofield, M. Y. Simmons, R. G. Clark, A. S. Dzurak, N. J. Curson, B. E. Kane, N. S. McAlpine, M. E. Hawley, and G. W. Brown, Phys. Rev. B 64, 161401(R) (2001).

[6] B. E. Kane, Nature (London) 393, 133 (1998)

[7] J. Kohler, Phys. Rep. 310, 261 (1999). 
[8] S. Ya. Kilin, A. P. Nizovtsev, T. M. Maevskaya, A. Drabenstedt, and J. Wrachtrup, J. Luminescence 86, 201 (2000).

[9] F. T. Charnock and T. A. Kennedy, Phys. Rev. B 64, 041201(R) (2001).

[10] A. M. Tyryshkin, S. A. Lyon, A. V. Astashkin, and A. M. Raitsimring, Phys. Rev. B 68, 193207 (2003).

[11] J. I. Cirac, P. Zoller, H. J. Kimble, and H. Mabuchi, Phys. Rev. Lett. 78, 3221 (1997).

[12] D. E. Browne, M. B. Plenio, S. F. Huelga, Phys. Rev. Lett. 91, 067901 (2003).

[13] S. Clark, A. Peng, M. Gu, and S. Parkins, Phys. Rev. Lett. 91, 177901 (2003).

[14] S. Mancini and S. Bose, Phys. Rev. A 70, 022307 (2004).

[15] L.-M. Duan, B. B. Blinov, D. L. Moehring, and C. Monroe, Quant. Inf. Comput. 4, 165 (2004).

[16] Y. L. Lim, A. Beige, and L. C. Kwek, Phys. Rev. Lett. 95, 030505 (2005).

[17] L.-M. Duan, B. Wang, H. J. Kimble, Phys. Rev. A 72, 032333 (2005).

[18] G. P. Berman, G. W. Brown, M. E. Hawley, D. I. Kamenev, and V. I. Tsifrinovich, quant-ph/0512174

[19] G. Feher, Phys. Rev. 114, 1219 (1959).

[20] G. P. Berman, G. D. Doolen, R. Mainieri, and V. I. Tsifrinovich, Introduction to Quantum Computers (World Scientific, Singapore, 1998).

[21] G. P. Berman, D. I. Kamenev, and V. I. Tsifrinovich, Perturbation Theory for Solid-State Quantum Computation with Many Quantum Bits (Rinton Press, Princeton, 2005).

[22] C. Herring, M. Flicker, Phys. Rev. 134, A362 (1964).

[23] G. P. Berman, G. D. Doolen, D. I. Kamenev, and V. I. Tsifrinovich, Phys. Rev. A 65, 012321 (2002). 